\title{
Competition or Cooperation Between Marine Biofilm Bacteria Recycling POM?
}

\author{
Kim. J. Everuss, Marina. W. Delpin and Amanda. E. Goodman
}

School of Biological Sciences, Flinders University, GPO Box 2100, Adelaide, South Australia 5001, Australia

Green fluorescent protein ( $g f p$ ) tagging has enabled the spatial analysis of different bacterial species within biofilms [1]. The use of GFP in conjunction with a Two-Photon Laser Scanning Microscope (TPLSM) facilitates the real time analysis of bacterial cells within a fully hydrated, living biofilm grown in a flow chamber [2]. Biofilms consist of vertically complex aggregates of microbial cells and secreted polymer attached to, and growing on, a surface or at an interface exposed to an aqueous phase [3].

This study investigated whether the chitinolytic marine bacterium Pseudoalteromonas sp. S91, which is able to completely hydrolyse squid pen can support Vibrio sp. S141 or Psychrobacter sp. SW5 in mixed species biofilms growing on squid pen in once through continuous flow chambers. Patterns of attachment on squid pen for gfp tagged Vibrio or gfp tagged Psychrobacter grown in monoculture were determined. Neither strain can grow on squid pen as the sole carbon source. Numbers of $g f p$ tagged cells in mixed species biofilms containing S91 were determined.

Images of the bacterial biofilms were collected using a Bio-Rad Radiance 2000MP visualising system in conjunction with a Nikon Eclipse TE300 inverted microscope. The microscope was equipped with a 60x water immersion lens with a numerical aperture of 1.2 and a Coherent Mira900-F titanium:sapphire ultrafast laser which has an excitation spectrum of $700-980 \mathrm{~nm}$. An excitation wavelength $(\lambda)$ at $800 \mathrm{~nm}$ was used with an emission $\lambda$ of $515-530 \mathrm{~nm}$, detected as green, for GFP visualisation and an emission $\lambda 450-480 \mathrm{~nm}$, detected as blue, for squid pen autofluorescence. Image analysis was performed using ImageJ 1.32 j [4] computer image analysis software. Numbers of $\mathrm{GFP}^{+}$bacteria in TPLSM images were quantified, essentially as done by Fitch et al. [5]; $\mathrm{GFP}^{+}$SW5, S91 and S141 were grown in liquid monoculture and separately viewed as wet mounts using the TPLSM.

This study showed that the motile marine bacterium S141, unable to efficiently metabolise squid pen, was supported by S91. After $72 \mathrm{~h}$, following various time periods of colonisation and growth within flow chambers (Fig. 1a), S141 cell numbers within dual species biofilms exhibited on average a 5-fold increase in the presence of S91. $\mathrm{GFP}^{+} \mathrm{S} 141$ cells were localised on the pen surface in monoculture. In the presence of S91, S141 cells were displaced or prompted to migrate away from the pen surface and attach to the vertically complex S91 biofilm (Fig. 1d).

In contrast, the marine bacterium SW5 was unable to be supported by S91 when grown in a dual species biofilm on squid pen. After 72 hours exposed to squid pen in monoculture, SW5 cells occurred singly and in small clusters in patches over the pen surface (Fig. 1b). In the presence of S91, SW5 cells were either absent or exhibited a significant decrease in cell numbers.

[1] A. Yoshida and H. K. Kuramitsu, Microbiology 148(Pt 11) (2002) 3385.

[2] S. Techkarnjanaruk et al., Appl Environ Microbiol. 63(8) (1997) 2989. 
[3] J. W. Costerton et al., Annu. Rev. Microbiol. 49 (1995) 711.

[4] W. Rasband, National Institutes of Health, USA.

[5] A. J.Fitch et al., Biofouling 18(4) (2002) 275.

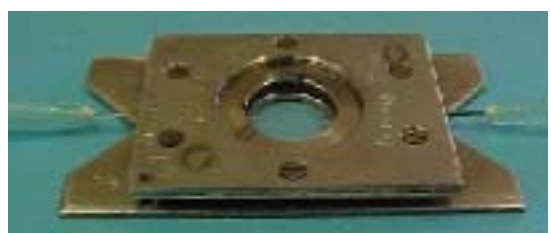

FIG. 1a. Metal flow chamber used to grow biofilms under flow conditions.

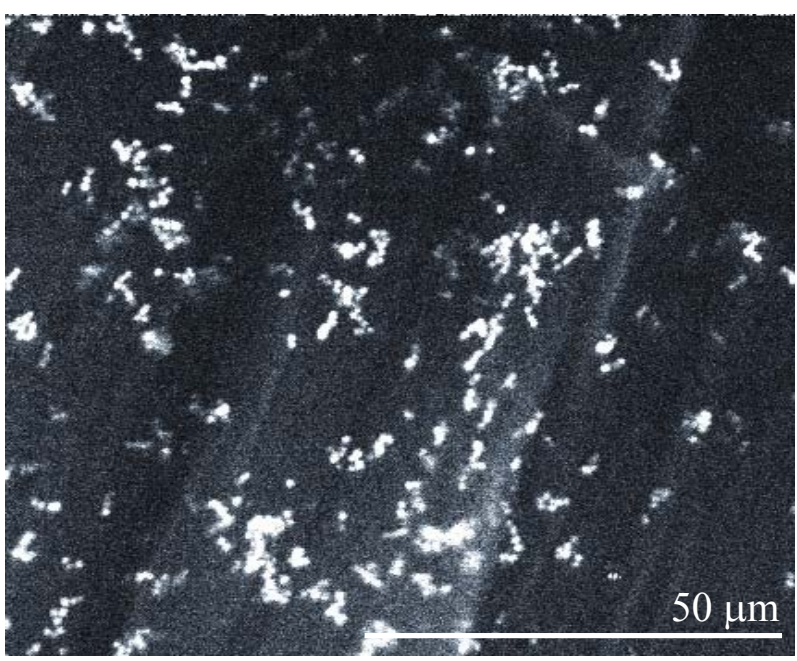

FIG. 1b. Green emission spectra $(515 \mathrm{~nm}-530 \mathrm{~nm}) x y$ image of SW5(GFP) cells on pen surface.

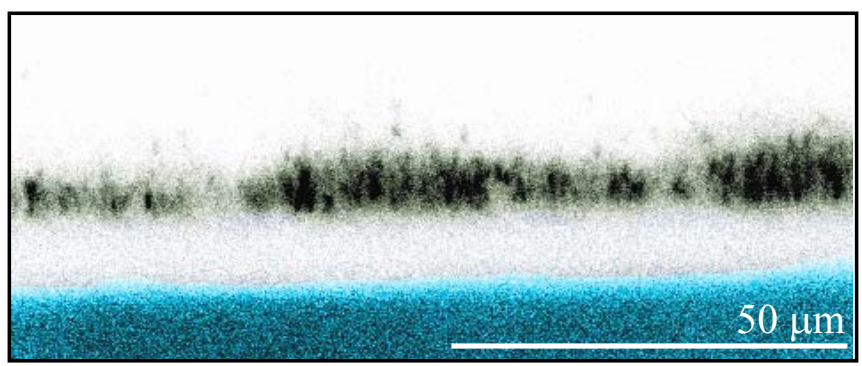

FIG. 1c. Green emission spectra $(515 \mathrm{~nm}-530 \mathrm{~nm}) x z$ image of S91(GFP) cells on pen surface (blue).

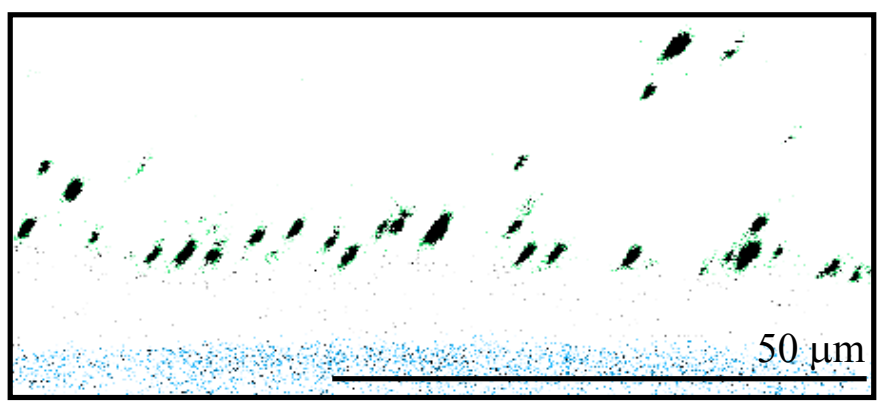

FIG. 1d. Green emission spectra $(515 \mathrm{~nm}-530 \mathrm{~nm}) x z$ image of S141(GFP) cells on pen surface (blue). 